\title{
Synthesis and Electrical Properties of Polyaniline Composite with Silver Nanoparticles
}

\author{
Safenaz M. Reda ${ }^{1 *}$, Sheikha M. Al-Ghannam ${ }^{2}$ \\ ${ }^{1}$ Chemistry Department, Faculty of Science, Benha University, Benha, Egypt \\ ${ }^{2}$ Chemistry Department, College of Girls for Science, University of Dammam, Dammam, KSA \\ Email: safenazr@yahoo.com
}

Received February 27, 2012; revised April 5, 2012; accepted April 15, 2012

\begin{abstract}
Polyaniline/silver (PANI/Ag) nanocomposite was prepared by chemical oxidative polymerization of aniline monomer in the presence of nitric acid. The formation of PANI/Ag nanocomposite was characterized by XRD, FTIR, TEM, UV-vis spectroscopy. The XRD patterns indicated that the crystalline phase of Ag is cubic with crystallite size of $93 \mathrm{~nm}$. The TEM image shows that the Ag nanoparticles are well dispersed in the polyaniline matrix. Optical measurements show that the value of optical band gap of nanocomposite is lower than that of pure PANI. The DC-, AC-conductivities, dielectric permittivity $\left(\varepsilon^{\prime}\right)$ and dielectric loss $\left(\varepsilon^{\prime}\right)$ of (PANI/Ag) nanocomposite and pure PANI have been measured in the temperature range from 303 to $723 \mathrm{~K}$ and frequency range from 10 to $10^{3} \mathrm{kHz}$. The electrical conductivity of the (PANI/Ag) nanocomposite is higher than pure PANI. Temperature variation of frequency exponents in this blend suggests that AC-conductivity is attributed to correlated barrier hopping mechanism. At all frequencies, the $\mathcal{E}^{\prime}$ value for (PANI/Ag) nanocomposite is higher than that for pure one. The higher dielectric constant of the PANI/Ag nanocomposite indicates their better ability to store electric potential energy under the influence of alternative electric field.
\end{abstract}

Keywords: Polyaniline/Silver (PANI/Ag) Nanocomposite; DC-Electrical Conductivity; AC-Electrical Conductivity; Dielectric Permittivity; Dielectric Loss

\section{Introduction}

Polymers are generally insulators and to exhibit electrical conductivity they must possess, ordered conjugation with extended (pi) electrons and large carrier concentrations. Conjugated polymers are the organic compounds that have an extended (pi) orbital system and conjugated carbon system [1]. Conductive polymer with polyaromatic backbone including polypyrrole, polythiophene, polyaniline, etc. has received a great deal of attention in the last two decades [2]. The conductivity of these conjugated polymers can be controlled by the process of doping which may be carried out through a chemical route, electrochemical route or photochemical route and is characterized by charge transfer from dopant to polymer or from polymer to dopant [1]. On doping these conjugated polymers show very high conductivity similar to metals. Therefore sometimes they are also called Synthetic Metals. They combine the electrical properties of metals with the advantage of polymers such as smaller weight, greater workability, resistance to corrosion and lower cost [1]. The most exciting applications of conductive polymers

*Present Address: Chemistry Department, College of Girls for Science, Dammam University, Dammam, Kingdom of Saudi Arabia. are in television sets, cellular telephones, automotive dashboard displays and artificial cockpit displays, Light emitting devices (LEDs), solar cells, lightweight batteries, light emitting diodes, polymer actuators, corrosion protection agents, sensors and molecular electronic devices $[1,2]$. Amongst the family of conducting polymers polyaniline (PANI) is one of the most promising electrically conducting polymers due to its unique electrical, electrochemical properties, easy polymerization, high environmental stability and low cost of monomer [3]. Also, its wide applications in microelectronic devices, diodes, light weight batteries, sensors, super capacitors, microwave absorption, corrosion inhibition [3]. Moreover, PANI has attracted attention for various reasons such as high absorption coefficients in the visible part of the spectrum, high mobility of charge carriers and tremendous stability [1]. Also PANI is unique among conductive polymers in that its electrical properties could be reversibly controlled both by charge transfer doping and by protonation, which makes it a potential material for applications such as chemical and biological sensors, actuators, microelectronic devices, etc. [4]. PANI exists in a number of forms which totally differ in chemical and physical properties. The most conducting emeraldine salt has conductivity on 
a semiconductor level of the order of $100 \mathrm{omh} \cdot \mathrm{cm}^{-1}$, many orders of magnitude higher than that of traditional polymers $\left(<10^{-9} \mathrm{omh} \cdot \mathrm{cm}^{-1}\right)$ but lower than that of metals $\left(>10^{4} \mathrm{omh} \cdot \mathrm{cm}^{-1}\right)$ [1]. PANI possesses secondary and tertiary amines in the backbone structure that can bind metal ions; these metal ions can be released by immersing inbound PANI into a low pH solution. The ability of binding metal ions and subsequent liberation makes PANI an attractive material for environmental safety [1]. However, PANI suffers from poor processability because it is infusible and insoluble in common solvents. The properties of PANI can be tailored by changing its oxidation states, dopants or through blending it with other organic, polymeric or inorganic nanosized semiconducting particles [3]. A number of metal and metal oxide particles have been encapsulated into the conductive polymer to form nanocomposites (NCs). The NCs exhibit combination of properties like conductivity, electrochemical, catalytic and optical properties [3]. The NCs are used in applications like electrochromic devices, light-emitting diodes, electromagnetic interference shielding, secondary batteries, electrostatic discharge systems, chemical and biochemical sensors [3]. To obtain materials with syncretistic advantage between PANI and inorganic nanoparticles, various composites of PANI with inorganic naonoparticles such as $\mathrm{Cu}$ [5], tellurium [6], lithium [7] and $\mathrm{TiO}_{2}$ [8] have been reported. No much work has been done on the polyaniline doped with noble metals such as silver (Ag). The incorporation of metal nanoparticles could effectively improve the electrical, optical and dielectric properties of the polyaniline composites [4]. These properties are extremely sensitive to small changes in the metal content and in the size and shape of the nanoparticles. It was reported that the nanoparticles themselves could act as conductive junctions between the PANI chains that resulted in an increase of the electrical conductivity of the composites [4]. The electrical conductivity of such composites might also depend upon the molecular structure of the conductive polymer matrix (i.e., crystallinity). Since silver exhibits the highest electrical and thermal conductivities among all the metals [4], the combination of PANI with silver could yield functional materials having enhanced electrical properties.

In this study, we explore the possibility of improving the conductivity of PANI by doping it with silver atoms. PANI/Ag NCs was prepared by chemical oxidation polymerization method under UV light. The electric and dielectric properties of the PANI/Ag NCs were investigated.

\section{Experimental}

\subsection{Synthesis}

Aniline (Aldrich) was dissolved in $1 \mathrm{M}$ nitric acid, as was silver nitrate (Aldrich). The solutions were mixed to start oxidation at room temperature. The concentration of aniline was $0.4 \mathrm{M}$, the silver nitrate-to-aniline molar ratio was 2:5. The reaction was slow, characterized by an induction period extending for weeks. So it was activated by exposing to UV light [9]. Green solids produced by the oxidation were collected on filter and washed several times by distilled water, and dried at room temperature.

The mechanism of synthesis of polyaniline-silver nanocomposite is given in Scheme 1. The amine group of aniline is transformed into protonated form by nitric acid. Nitric acid capped silver nanoparticles have negative charges on their surface. Electron negative silver nanoparticles adsorb the positively charged aniline cations through electrostatic interactions prior to polymerization and form aniline-silver complex [9].

The DC-, AC-conductivity, dielectric constant $\varepsilon^{\prime}$ and dielectric loss $\varepsilon^{\prime \prime}$ as a function of temperature and frequency were measured. This was carried out using programmable automatic LCR meter (PM636 Philips) at frequencies of $10-10^{3} \mathrm{kHz}$ and temperature range of 303 $-723 \mathrm{~K}$.

\subsection{Characterization}

Structure characterization of the prepared polyanilinesilver composite was carried out by the X-ray diffraction (XRD) using Diano Corporation USA diffractometer with the Co-radiation $(\lambda=0.179 \mathrm{~nm})$. The optical absorbance was examined by using a Perkin-Elmer Lambda 40 spectrophotometer over the spectrum range of 200 $800 \mathrm{~nm}$ at room temperature. A transmission electron microscopy (TEM) examination was made to observe the surface morphology of samples using an EM10 Zeiss West Germany electron probe microanalyzer. The infrared absorption (IR) spectra, between 4000 and $400 \mathrm{~cm}^{-1}$, were obtained using a Nicolet spectrometer model 670 FT-IR on $\mathrm{KBr}$ pellet of the samples.

\section{Results and Discussion}

\subsection{Characterization}

\subsubsection{XRD Study}

XRD patterns of the synthesized polyaniline-silver composite are shown in Figure 1. The diffraction peaks at $2 \theta$ $=\left(38.3^{\circ}\right),\left(44.4^{\circ}\right),\left(64.6^{\circ}\right)$ and $\left(77.5^{\circ}\right)$ to the $(111),(200)$, (220) and (311) diffraction planes, respectively, ascribed to the cubic structure of $\mathrm{Ag}$ [2].

The average crystallite size of the polyaniline-silver composite was calculated using the Scherrer Equation [10]:

$$
D=\frac{0.94 \lambda}{\beta \cos \theta}
$$

where $D$ is the crystal size of $\mathrm{Ag}, \lambda$ the wavelength of 
X-ray $(0.179 \mathrm{~nm}), \theta$ half diffraction angle of peak (in degrees) and $\beta$ the true half peak width. The average size of the Ag determined through the (111) plane is $93 \mathrm{~nm}$.

\subsubsection{FT-IR Study}

Figure 2 represents the FT-IR spectra of PANI/Ag. The bands at 1300,1490,1250,1370, 1030 and $806 \mathrm{~cm}^{-1}$ correspond to polyaniline. The band corresponding to out of plane bending vibration of $\mathrm{C}-\mathrm{H}$ bond of p-substituted benzene ring appears at $806 \mathrm{~cm}^{-1}$. The bands corresponding to stretching vibrations of $\mathrm{N}-\mathrm{B}-\mathrm{N}$ and $\mathrm{N}=\mathrm{Q}=\mathrm{N}$ structures appear at 1490 and $1600 \mathrm{~cm}^{-1}$, respectively, (where - $\mathrm{B}$ - and $=\mathrm{Q}=$ stand for benzenoid and quinoid moieties in the polyaniline backbone) [2]. The peak appeared at 1170 $\mathrm{cm}^{-1}$ corresponds to $-\mathrm{N}=\mathrm{Q}-\mathrm{N}^{+}-\mathrm{B}$ - which is characteristic of the protonated state. The absorption band at $1250 \mathrm{~cm}^{-1}$ is associated with polaronic structure of PANI [2]. The

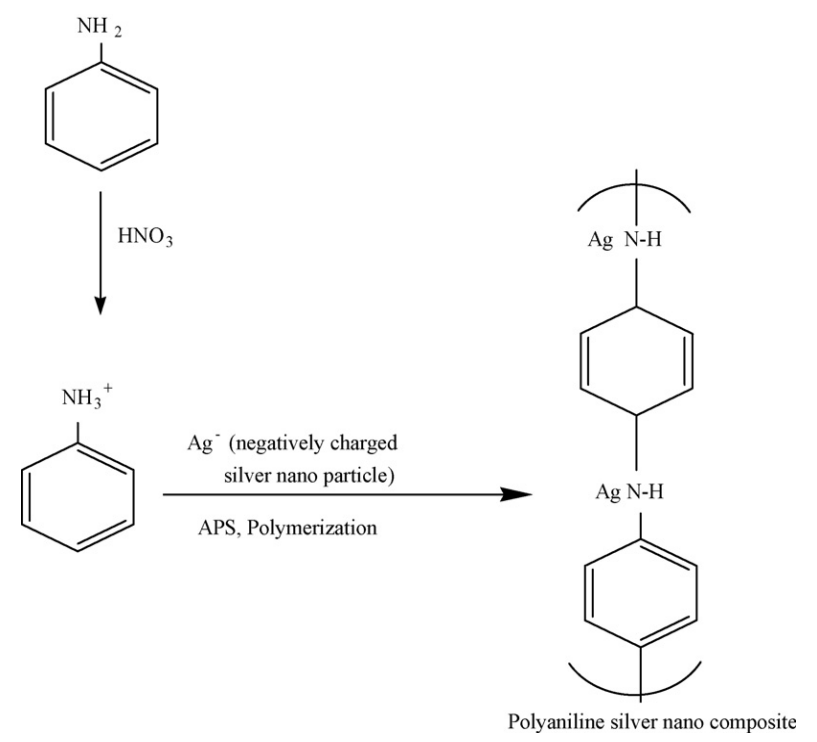

Scheme 1. Mechanism of formation of polyaniline-silver nanocomposite.

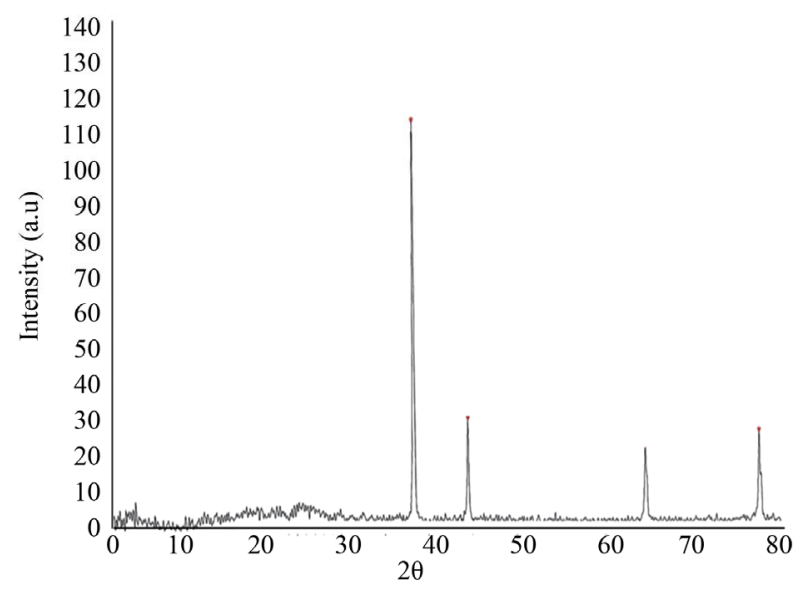

Figure 1. X-ray diffraction patterns of PANI/Ag nanocomposite.

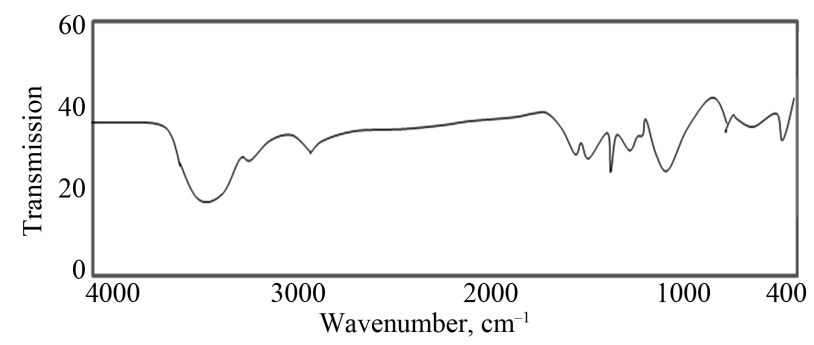

Figure 2. FTIR spectrum of PANI/Ag nanocomposite.

bands at $1146 \mathrm{~cm}^{-1}$ correspond to polyaniline in the composites. The bands corresponding to vibration mode of $\mathrm{N}=\mathrm{Q}=\mathrm{N}$ ring and stretching mode of $\mathrm{C}-\mathrm{N}$ bond appear at 1146 and $1300 \mathrm{~cm}^{-1}$, respectively.

\subsubsection{Morphological Study}

Figures 3(a) and (b) shows TEM images for the PANI and the PANI/Ag nanocomposite, respectively. TEM shows that the samples consist of nanowires and nanorods, with width gradually decreasing from bottom to top the tip. It was found that $\mathrm{Ag}$ has a strong effect on the PANI's morphology; it shows a transformation in morphology of PANI particles. As shown in TEM images (Figure 3(b)), the silver particles are well adhered on the PANI substrate due to strong affinity of silver to nitrogen. This indicates that nano-sized inorganic particles possess a nearly spherical morphology and influence strongly the composite morphology. Since the silver nanoparticles were synthesized in the polyaniline solution, the nanoparticles got embedded into the polyaniline matrix [2]. It can also be seen that the spherically shaped Ag nanoparticles are uniformly dispersed in the PANI matrix and the particles are not clearly monodispersed. From the TEM

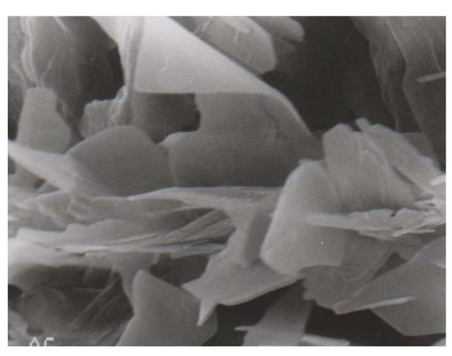

(a)

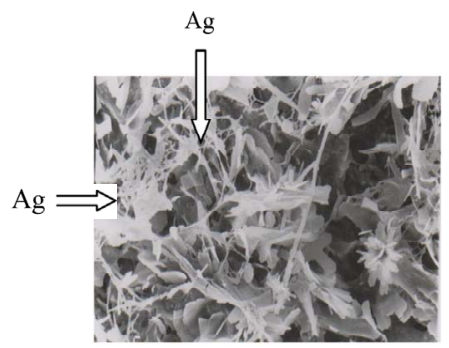

(b)

Figure 3. TEM of PANI (a) and PANI/Ag nanocompsite (b). 
image it is observed that grains are well resolved and circular in shape and dopant particles are uniformly distributed in the polymer matrix. The formation of a relatively large cluster with Ag dispersity could be attributed to silver migration and aggregation. The migration and aggregation of silver particles might be driven by the instability of silver atoms due to their high surface free energy. Their aggregation would produce thermodynamically stable clusters [2].

\subsubsection{UV-Visible Absorption Study}

The photon absorption in many amorphous materials is found to obey the Tauc's relation [2]:

$$
(\alpha h v)=\left(h v-\mathrm{E}_{\mathrm{g}}\right)^{\mathrm{n}}
$$

where $\left(E_{g}\right)$ the optical band gap, $(\alpha)$ the absorption coefficient, and $(h v)$ the energy of the incident photon. The index $\mathrm{n}$ has discrete values such as $1 / 2,3 / 2,2$ or higher depending on whether the transition is direct or indirect and allowed or forbidden. In the direct and allowed cases, the index $n$ is $1 / 2$, whereas for the direct but forbidden cases it is $3 / 2$. But for the indirect and allowed cases $n=$ 2 and for the forbidden cases it is 3 or higher. In the present case the photon energy $(h v)$ is plotted against $(\alpha h v)^{2}$ for $\mathrm{n}=0.5$, Figure 4. It gives a straight line fit, which implies that the samples undergo direct transition. Then the band gap has been extracted by extrapolating the straight portion of the graph on $h v$ axis at $\alpha=0$. The calculated values of the optical band gap for PANI and PANI/Ag are 2.7 and $2.25 \mathrm{eV}$, respectively. The value of optical band gap is found to decrease after doping. Since the optical absorption depends on the short range order in the amorphous state and defect state associated with it, the decrease in the optical band gap in the present system may be due to reduction in the disorder of the system and increase in the density of defect states. From this it is clear that the silver doping has a potential effect on the optical properties of PANI. Optical conductivity of polyaniline increases in presence of silver nanoparticles due to the decrease in optical band gap and this type of behavior is also observed in the electrical conductivity measurements.

\subsection{DC-Electrical Conductivity}

To illustrate the effect of Ag nanoparticles on the DCconductivity of polyaniline material, a comparison of pure PANI and PANI/Ag nanocomposite was made. Figure 5 shows the temperature dependence of DC-conductivity in the temperature range 303 - $723 \mathrm{~K}$ for undoped PANI and PANI/Ag. From the figure, it is evident that the DC-electrical conductivity of the composite is higher than that of polyaniline. It is clear from the figure that in all the samples, the plots of $\ln \sigma_{\mathrm{DC}}$ vs $1000 / \mathrm{T}$ are nearly straight lines, indicating that the conduction in these samples through an activated process having single activation energy in the temperature range $303-723 \mathrm{~K}$. The activation behavior of the samples are studied by using Arrhenius Equation [1],

$$
\sigma_{\mathrm{DC}}=\sigma_{\mathrm{O}} e^{\frac{-\mathrm{E}_{\mathrm{a}}}{2 k \mathrm{~T}}}
$$

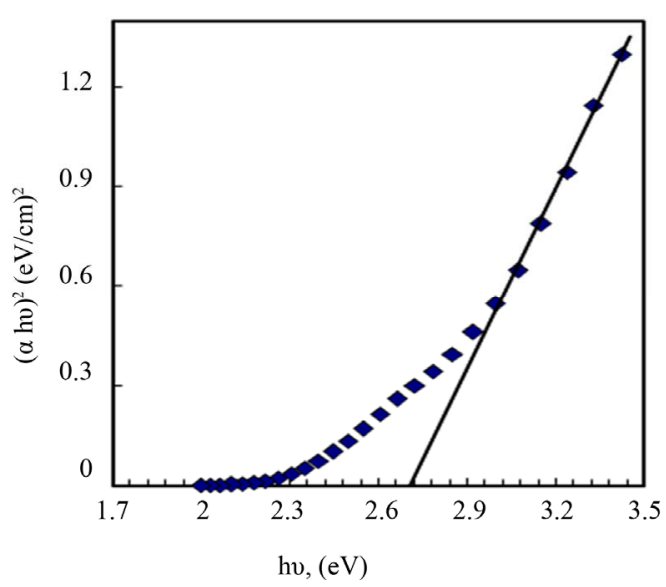

(a)

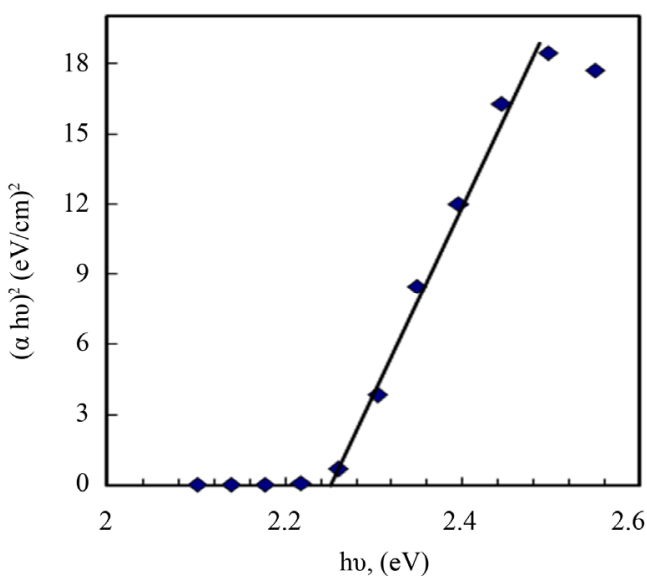

(b)

Figure 4. Determination of band gap energy for PANI (a) and PANI/Ag nanocomposite (b).

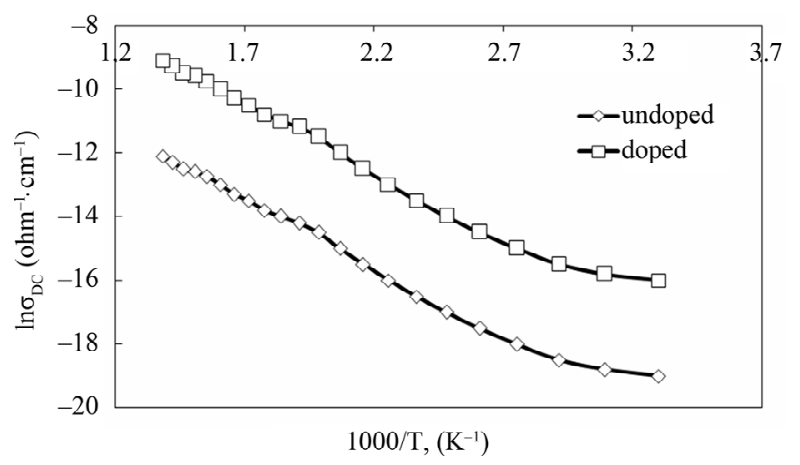

Figure 5. Effect of temperature on DC-electrical conductivity for all investigated samples. 
where $k$ is the Boltzman's constant and $\sigma_{\mathrm{O}}$ is the conductivity at infinity temperature.

The values of activation energy calculated from Figure 5 and are given in Table 1. It was found that the activation energy for pure PANI is higher than PANI/Ag composite. An increase in DC-conductivity with corresponding decrease in activation energy is found to be associated with a shift of Fermi level in doped samples [1]. From a single value of activation energy it is clear that the conduction is through the carrier concentration at the Fermi level. But the activation energy $\left(\mathrm{E}_{\mathrm{a}}\right)$ alone does not provide any information whether the conduction takes place in extended states or by hopping in localized states. This can be explained on the basis of the values of pre-exponential factor $\left(\sigma_{\mathrm{o}}\right)$. According to Mott and Davis [11] a value of $\sigma_{\mathrm{o}}$ in the range $10^{3}-10^{4} \mathrm{ohm} \cdot \mathrm{cm}^{-1}$ indicates that the conduction takes place mostly in extended states. A smaller value of $\sigma_{\mathrm{o}}$ indicates a wide range of localized states and the conduction is taking place by the hopping process. In our case, the values of $\sigma_{\mathrm{o}}$ are found to be of the order of $10^{-3}$ for PANI and $10^{-2}$ for PANI/Ag, therefore the conduction takes place by the hopping process due to the wide range of localized states present in the sample. From the above results we can conclude that the hopping mechanism is responsible for an increase in the conductivity of the samples. A smaller value of $\sigma_{\mathrm{o}}$ indicates that the density of defect states increases in the sample and further supports our argument that the conduction mostly takes place by the hopping process in the Ag doped polyaniline. The formation of polarons and bipolarons can also be used to explain the conduction mechanism [1]. Polarons and bipolarons play a leading role in determining the charge injection, optical and transport properties of conductive polymers. These are self-localized particles like defects associated with characteristic distortions of the polymer backbone and with quantum states deep in the energy gap due to strong lattice coupling.

\subsection{AC-Electrical Conductivity}

The frequency-temperature dependences of AC-electrical conductivity of PANI and PANI/Ag nanocomposite are shown in Figure 6. As shown in this figure, the AC-electrical conductivity of the PANI/Ag nanocomposite is higher than that of the pure PANI. The lower conductivity of the pure PANI could be ascribed to low level of protonation of the PANI chains. The improvement of ACconductivity for PANI/Ag nanocomposite comes from the effective dispersion of $\mathrm{Ag}$ nanoparticles in the PANI matrix (shown in TEM images), which favors better electronic transport. It is evident that the AC-conductivity is both frequency and temperature dependent and enhanced with an increase of both the frequency and the temperature. This indicates that there may be charge carriers, which can be transported by hopping through the defect sites along the polymer chain [4].

It can be seen that the conductivity of PANI/Ag nanocomposite increases as the frequency increases. The frequency dependence of the AC-conductivity is considered to be a result of interface charge polarization (or Maxwell-Wagner-Sillars effect) and intrinsic electric dipole polarization [4]. This phenomenon appears in heterogeneous systems like metal-polymer composites due to the accumulation of mobile charges at the interfaces and the formation of large dipoles on metal particles or clusters. Several theoretical models have been proposed for the AC-conduction in amorphous semiconductors such as classical hopping and quantum mechanical tunneling of

Table 1. DC- and AC-conductivity data for all investigated samples.

\begin{tabular}{cccccc}
\hline Samples & $\begin{array}{c}\mathrm{E}_{\mathrm{a}} \\
(\mathrm{eV})\end{array}$ & $\begin{array}{c}{ }^{*} \text { Temp. } \\
\text { Range } \\
(\mathrm{K})\end{array}$ & $\begin{array}{c}{ }^{\mathrm{S}} \sigma_{\mathrm{DC}} \\
\mathrm{ohm}^{-1} \cdot \mathrm{cm}^{-1}\end{array}$ & $\begin{array}{c}\mathrm{W}_{\mathrm{M}} \times 10^{3} \\
(\mathrm{eV})\end{array}$ & $\begin{array}{c}{ }^{*} \sigma_{\mathrm{AC}} \\
\mathrm{ohm}^{-1} \cdot \mathrm{cm}^{-1}\end{array}$ \\
\hline PANI & 0.32 & $370-450$ & $3.1 \times 10^{-6}$ & 0.25 & $3.3 \times 10^{-3}$ \\
PANI/Ag & 0.10 & $370-450$ & $6.1 \times 10^{-5}$ & 0.01 & $5.2 \times 10^{-2}$ \\
\hline
\end{tabular}

${ }^{\$} \sigma_{\mathrm{DC}}$ at $563 \mathrm{~K} ;{ }^{*} \sigma_{\mathrm{AC}}$ at $563 \mathrm{~K}$ and frequency of $100 \mathrm{kHz} ;{ }^{\#}$ Temperature range at which the activation energy; $\mathrm{E}_{\mathrm{a}}$, was calculated.

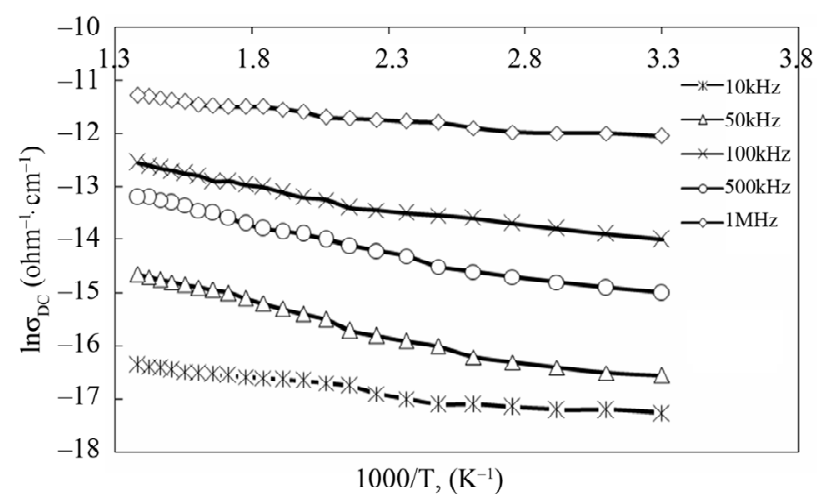

(a)

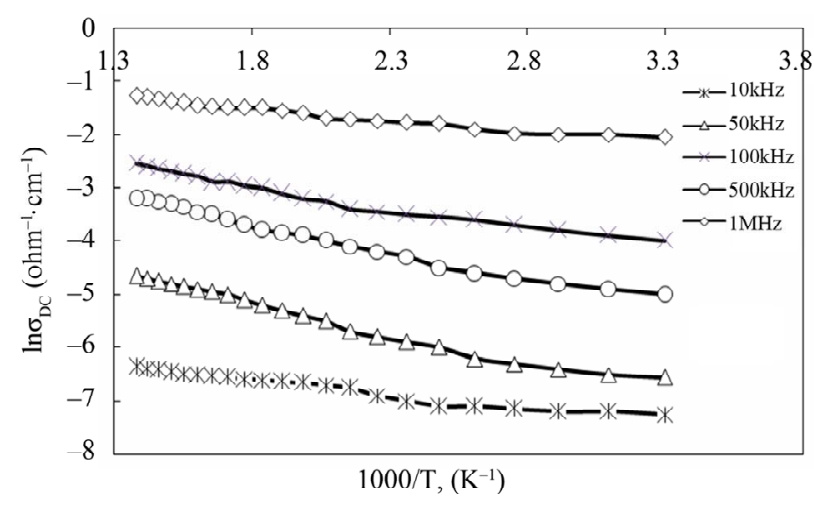

(b)

Figure 6. Effect of temperature on AC-electrical conductivity for PANI (a) and PANI/Ag nanocomposite(b). 
charge carriers over the potential barrier separating two energetically favorable centers in a random distribution [12]. In electron tunneling model, $\mathrm{s}$ is independent of $\mathrm{T}$. In case of small polaron tunneling, $\mathrm{s}$ decreases as $\mathrm{T}$ increases. The variation of $\mathrm{s}$ with $\mathrm{T}$ for the PANI/Ag is shown in Figure 7, where a decrease in the values of $s$ becomes higher as the temperature is increased. In the correlated barrier hopping $(\mathrm{CBH})$ model, the charge carrier hops between the sites over the potential barrier separating them. The existence of Coulomb interaction between individual barriers causes a barrier reduction. The frequency exponent $\mathrm{s}$ for such model is given by the following Equation [12]:

$$
S=1-\left\{\frac{6 k T}{W_{M}}\right\}
$$

where $W_{M}$ is the effective barrier height at infinite interstice separation R. From Table 1, it can be seen that the highest values of $W_{M}$ are assigned to PANI compared with the PANI/Ag. The high potential barrier exhibited in this sample acts to hinder charge carrier transport and this is responsible for its lower conductivity.

\subsection{Temperature Dependence of Dielectric Permittivity and Dielectric Loss}

Figures 8 and 9 show the temperature dependence of the dielectric constants and dielectric losses of PNAI and $\mathrm{PNAI} / \mathrm{Ag}$ in the frequency range of $10-10^{3} \mathrm{kHz}$ and temperature range of 303 - $723 \mathrm{~K}$. In these figures, higher dielectric constants and losses of PANI/Ag nanocomposite than those of pure PANI are observed. It can be seen that the incorporation of Ag enhanced the dielectric constant of the PANI/Ag nanocomposite about 3 times of that recorded for the pure PANI under the same conditions, Figure 8. At the same time, there is about a four orders of magnitude increase in dielectric loss upon incorporation of $\mathrm{Ag}$ in the polyaniline, Figure 9. From the results described above, it has been conclude that the enhanced dielectric constants and losses might originate from the increased conductivity. The better dielectric

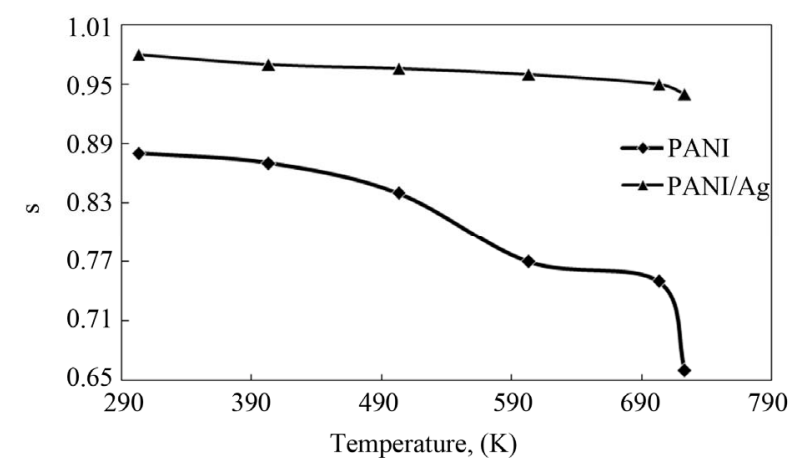

Figure 7. Effect of temperature on s value for all investigated samples. constant of the PANI/Ag nanocomposite indicates their better ability to store electric potential energy under the influence of alternative electric field. The frequency dependency of dielectric constant is found to be increased in presence of $\mathrm{Ag}$ in the composite (Figure 8). This is thought to be a result of the slow dielectric relaxation of the PANI matrix and the interface of the composite. The dielectric constant and loss are very high due to the AC-conduction loss that dominates over other types of losses present in the polymer e.g. dipole segmental losses and orientation of the polar group [4].

\section{Conclusion}

We have synthesized polyaniline and polyaniline-silver nanocomposite by chemical oxidative method. The formation of Ag nanoparticles and their presence in the prepared nanocomposite were confirmed by XRD, FTIR, TEM, UV-Vis spectroscopy. The XRD patterns indicated that the crystalline phase of $\mathrm{Ag}$ is cubic with crystallite size of $93 \mathrm{~nm}$. TEM analysis showed uniform dispersion of the spherically shaped Ag nanoparticles in the PANI matrix. The optical studies indicated that the absorption mechanism is due to direct allowed transition and the optical band gap of polyaniline is higher than that of

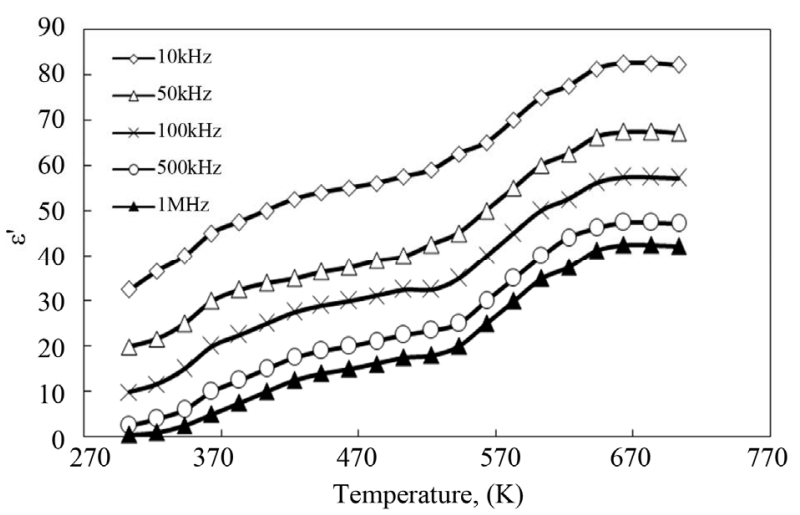

(a)

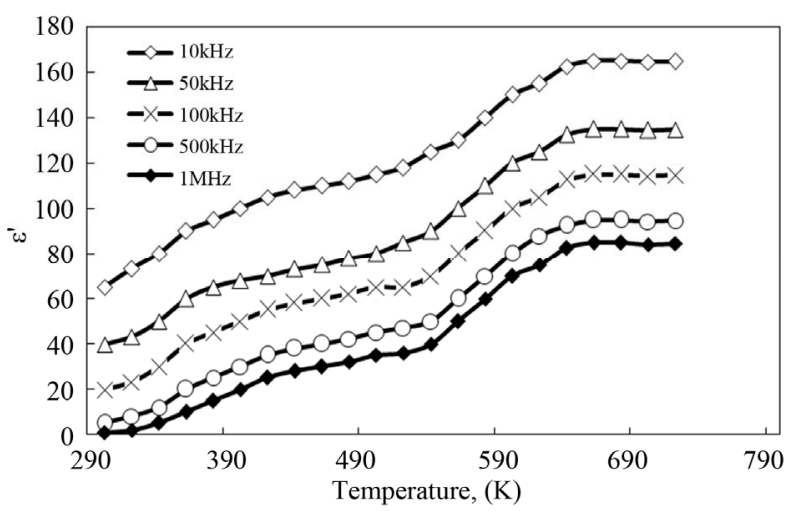

(b)

Figure 8. Effect of temperature on dielectric constant for PANI (a) and PANI/Ag nanocomposite (b). 


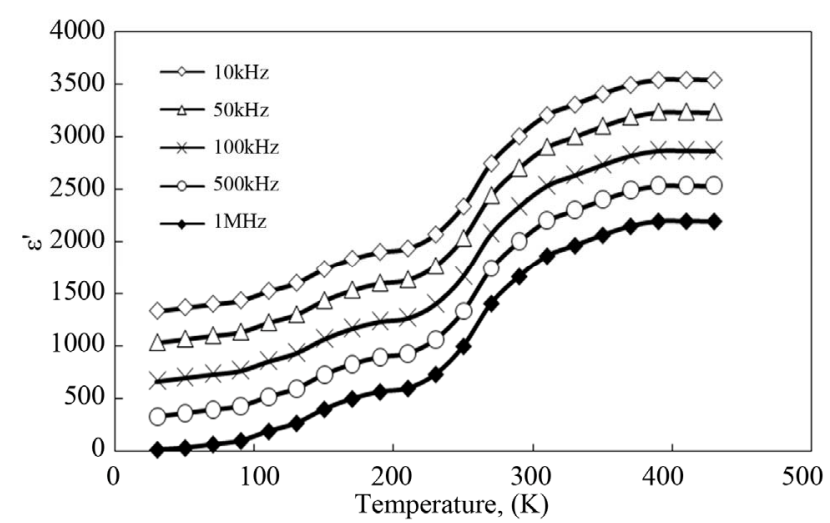

(a)

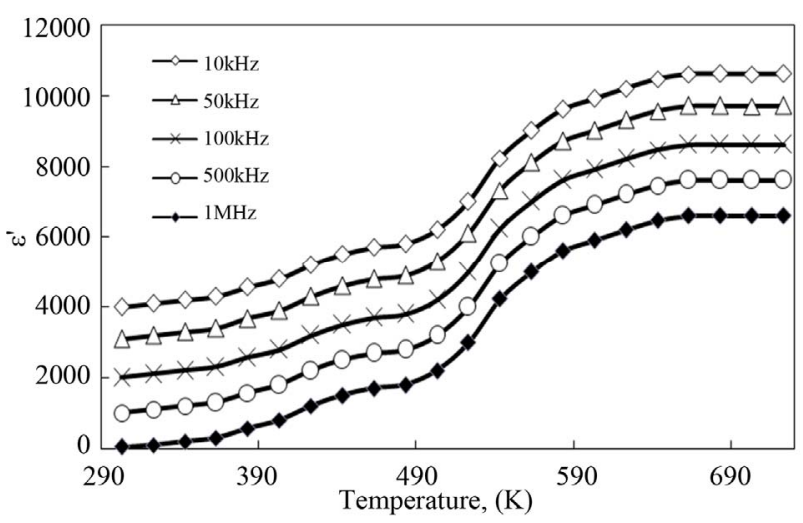

(b)

Figure 9. Effect of temperature on dielectric loss for PANI (a) and PANI/Ag nanocomposite (b).

polyaniline/silver (PANI/Ag) nanocomposite. Conductivity and dielectric properties such as dielectric constant and dielectric loss have been measured and the conduction mechanism has also been investigated in terms of Mott and Davis. It is suggested that conduction is taking place by the hopping process due to a wide range of localized states present in the sample. The PANI/Ag nanocomposite exhibit remarkable improvement of electrical conductivity and dielectric properties when compared with pure PANI. So, this is a simple way by which optical and electrical properties of other conductive polymers may be enhanced by using different nanoparticles.

\section{Acknowledgements}

The authors wish to gratefully acknowledge the support from Chemistry Department, College of Science for Girls, Dammam University, Dammam, Kingdom of Saudi Arabia.

\section{REFERENCES}

[1] G. B. Shumaila, V. S. Lakshmi, M. Alam, A. M. Siddiqui, M. Zulfequar and M. Husain, "Synthesis and Characterization of Se Doped Polyaniline," Current Applied Physics, Vol. 11, No. 2, 2010, pp. 217-222.

doi:10.1016/j.cap.2010.07.010

[2] K. Gupta, P. C. Jana and A. K. Meikap, "Optical and Electrical Transport Properties of Polyaniline-Silver Nanocomposite," Synthetic Metals, Vol. 160, No. 13-14, 2010, pp. 1566-1573.

[3] S. S. Umare, B. H. Shambharkar and R. S. Ningthoujam, "Synthesis and Characterization of Polyaniline- $\mathrm{Fe}_{3} \mathrm{O}_{4} \mathrm{Na}-$ nocomposite: Electrical Conductivity, Magnetic, Electrochemical Studies," Synthetic Metals, Vol. 160, No. 17-18, 2010, pp. 1815-1821. doi:10.1016/j.synthmet.2010.06.015

[4] A. Choudhury, "Polyaniline/Silver Nanocomposites: Dielectric Properties and Ethanol Vapour Sensitivity," Sensors and Actuators B: Chemical, Vol. 138, No. 1, 2009, pp. 318-325. doi:10.1016/j.snb.2009.01.019

[5] V. Ali, R. Kaur, N. Kamal, S. Singh, S. C. Jain, H. P. S. Kang, M. Zulfequar and M. Husain, "Use of $\mathrm{Cu}^{+1}$ Dopant and It's Doping Effects on Polyaniline Conducting System in Water and Tetrahydrofuran," Journal of Physics and Chemistry of Solids, Vol. 67, No. 4, 2006, pp. 659664. doi:10.1016/j.jpcs.2005.10.172

[6] S. Kazim, V. Ali, M. Zulfequar, M. M. Haq and M. Husain, "Electrical, Thermal and Spectroscopic Studies of Te Doped Polyaniline," Current Applied Physics, Vol. 7, No. 1, 2007, pp. 68-75. doi:10.1016/j.cap.2005.11.072

[7] K. S. Ryu, B. W. Moon, J. Joo and S. H. Chang, "Characterization of Highly Conducting Lithium Salt Doped Polyaniline Films Prepared from Polymer Solution," Polymer, Vol. 42, No. 23, 2001, pp. 9355-9360. doi:10.1016/S0032-3861(01)00522-5

[8] J. C. Xu, W. M. Liu and H. L. Li, "Titanium Dioxide Doped Polyaniline," Material Science Engineering: C, Vol. 25, No. 4, 2005, pp. 444-447.

[9] N. V. Blinova, J. Stejskal, M. Trchova, I. Sapurina and G. C. Marjanovic, "The Oxidation of Aniline with Silver Nitrate to Polyaniline-Silver Composites," Polymer, Vol. 50, No. 1, 2009, pp. 50-56. doi:10.1016/j.polymer.2008.10.040

[10] M. Ristić, M. Ivanda, S. Popvić and S. Musić, "Dependence of Nanocrystalline $\mathrm{SnO}_{2}$ Particle Size on Synthesis Route," Journal of Non-Crystalline Solids, Vol. 303, No. 2, 2002, pp. 270-280. doi:10.1016/S0022-3093(02)00944-4

[11] N. F. Mott and E. A. Davis, "Electronic Process in NonCrystalline Materials," Clarendon Press, Oxford, 1979.

[12] S. Ebrahim, A. H. Kashyout and M. Soliman, "Ac and Dc Conductivities of Polyaniline/PolyVinyl Formal Blend Films," Current Applied Physics, Vol. 9, No. 2, 2009, pp. 448-454. doi:10.1016/j.cap.2008.04.007 